\title{
Random Access Over Multiple Access Channels: A Queuing Perspective
}

\author{
Shreeshankar Bodas, Sriram Vishwanath \\ The University of Texas at Austin \\ \{bodas, sriram\}@ece.utexas.edu
}

\author{
Vijay Subramanian \\ Hamilton Institute, Ireland \\ Vijay.Subramanian@nuim.ie
}

\begin{abstract}
This paper investigates a slotted random access system where packet capture is modeled using communication theoretic techniques. It is shown that the optimum rates and attempt probabilities depend on the receiver architecture, operating signal-to-noise ratio (SNR) and block length. High SNR analysis reveals that joint decoder asymptotically yields either a collision channel or a non-interacting channel, and single-user decoder results in a collision channel. Specific scenarios are investigated at intermediate SNR to determine when this all-or-nothing regime applies.
\end{abstract}

\section{INTRODUCTION}

Random access multiple access or broadcast channels with different physical layer parameters have been well studied in literature [9] [10] [6] [5] [2] [3]. Traditional analysis of stability of such systems assumes a relatively simplistic capture model [8], or leaves it as a parameter to be determined by the physical layer [7]. On the flip side, traditional information theoretic techniques assume "perfect capture", indicating that, at the appropriate pre-determined rates, both users must transmit simultaneously for arbitrarily long block-lengths. For practical block-lengths and realistic probabilities of error, it is intuitively better for users not to collide, but the "collision causes full loss" assumption is high unrealistic, given all the advancements in receiver architectures, especially for MIMO systems.

In this paper, we analyze the the behavior of the ALOHA protocol in the context of multiple access channel. We consider two regimes - high signal-to-noise ratio (SNR) and intermediate SNR, and different kinds of decoder - single user decoder, successive interference cancellation decoder and multi-user (i.e., joint) decoder. We show that, depending upon the SNR and the type of decoder, the probability of transmission attempt that maximizes the per-user throughput can lie between 0 and 1 , i.e., it is not always beneficial for both users to transmit simultaneously.

The rest of this paper is organized as follows: In Section II, we present the channel and queuing model. In Section III, we focus on the case of high SNR and derive expressions for the optimal probability of transmission attempt for different decoders. In Section IV, we analyze the system with an intermediate value of SNR. We conclude in Section V, and numerical results in the Appendix.

This work is supported in part by an ARO Young Investigator Award, NSF Grants CNS-0626903 and CCF-0634898, and Science Foundation of Ireland Grant 07/IN.1/I901.

\section{SySTEM MODEL}

\section{A. Channel Model}

We consider the multiple access channel described in [11]. There are 2 non-co-operating users who want to transmit data to a single receiver. Each transmitter has $m$ antennas, and the receiver has $n$ antennas. The transmitted signal from each user to the receiver undergoes Rayleigh fading, which remains constant over a block of $\ell \geq 2 m+n-1$ transmit symbols (slow fading). The fading coefficients are known at the receiver, while the transmitters only know the statistics of the respective channels and not the realizations. Further, the received signal is corrupted by additive complex Gaussian noise. The noise at each of the receive antennas at each time is i.i.d. $\mathcal{C N}(0,1)$, where $\mathcal{C N}(0, a)$ denotes a complex Gaussian random variable with i.i.d. zero mean, variance $a / 2$, Gaussian random variables as its real and imaginary parts. SNR denotes the ratio of the user's transmit power to the (Gaussian) noise power at the receiver. Thus, the received signal at the end of $\ell$ time-slots is

$$
\mathbf{Y}=\sqrt{\frac{\mathrm{SNR}}{m}} \sum_{i=1}^{2} \mathbf{H}_{i} \mathbf{X}_{i}+\mathbf{W} .
$$

The fading coefficients matrix $\left\{\mathbf{H}_{i}\right\}_{i=1,2}$ models the slow fading channels. The entries of $\left\{\mathbf{H}_{i}\right\}_{i=1,2}$ are i.i.d., $\mathcal{C N}(0,1)$.

\section{B. Queuing Model}

We consider a 2-user multiple access channel as shown in Figure 1. The two users, $A$ and $B$, each serve a queue with input rate $\Lambda_{A}(\mathrm{SNR})=\lambda_{A} \log \mathrm{SNR}$ and $\Lambda_{B}(\mathrm{SNR})=$ $\lambda_{B} \log$ SNR bits per transmission slot, respectively. We assume that each transmission slot is $l$ transmit symbols long, i.e., the same duration as the channel coherence time. When the queue is nonempty, each user selects a packet uniformly at random from the queue and attempts transmission, with probability $p_{i}, i=A, B$, and independently of the other user. Each user transmits at rate $R_{i}(\mathrm{SNR})=r_{i} \log \mathrm{SNR}, i=A, B$. Depending upon the channel realization, the type of decoder, the transmission rate and whether the other user attempts transmission, a user's transmission is successfully decoded by the receiver $C$ with a certain probability. There is instantaneous feedback from the receiver to the respective transmitters which enables them to delete successfully received packets from their queues and retain those packets whose transmission has failed. We derive the capacity region for the 2-user MAC, i.e., all pairs $\left(\lambda_{A}, \lambda_{B}\right)$ such that the queues are stable. Thereafter the aim is 
to obtain the value of the attempt probabilities $p_{i}, i=A, B$ for the max-min fair operating point ([1], Section 4.2). We study the system in the following regimes:

- High SNR, joint (optimal) decoder at the receiver,

- High SNR, single user decoder at the receiver,

- High SNR, successive interference cancellation decoder,

- Intermediate value of SNR, single-user decoder at the receiver.

The first two decoders represent the two extremes of possible decoders: one is complex to implement but gives better performance in terms of throughput, while the other is simpler to implement but has poorer throughput. The last two regimes are of interest for practical scenarios.

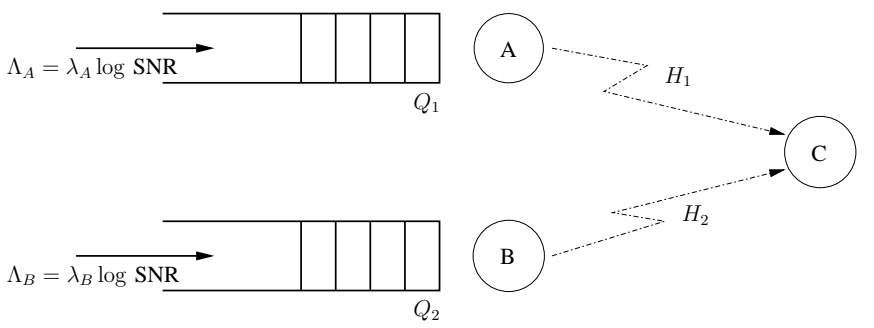

Fig. 1. The System Model - Multiple Access Channel

\section{THE HIGH SNR REGIME}

In order to analyze the system throughput at high values of SNR, we start with a definition and an important result from [11].

Definition 1. A scheme $\{C(S N R)\}$ is said to achieve spatial multiplexing gain $r$ and diversity gain $d$ if the data rate $R(S N R)$ and the average probability of error $P_{e}(S N R)$ satisfy

and

$$
\lim _{S N R \rightarrow \infty} \frac{R(S N R)}{\log S N R} \geq r
$$

$$
\lim _{S N R \rightarrow \infty} \frac{\log P_{e}(S N R)}{\log S N R} \leq-d .
$$

Fact 1: ([12], Thm. 2) For a point-to-point channel with blocklength $\ell \geq m+n-1$, the optimal diversity-multiplexing tradeoff curve $\left(r, d^{*}(r)\right)$ is given by the piecewise linear function joining the points $(k,(m-k)(n-k)), 1 \leq k \leq \min (m, n)$.

Fact 2: ([11], Thm. 3) For a multiple access channel with $K$ users and block-length $\ell \geq K m+n-1$, when all the users transmit at the same rate $r$ (symmetric case), the optimal diversity function $d_{\text {sym }}^{*}(r)$ is given by

$$
d_{\text {sym }}^{*}(r)= \begin{cases}d_{m, n}^{*}(r), & r \leq \min \left(m, \frac{n}{K+1}\right), \\ d_{K m, n}^{*}(K r), & r \geq \min \left(m, \frac{n}{K+1}\right),\end{cases}
$$

where $d_{m, n}^{*}(r)$ denotes the optimal diversity function for a point-to-point channel with $m$ transmit antennas and $n$ receive antennas. Instead if the users transmit at rates $r_{k}, k=$ $1,2, \ldots, K$, then the maximum diversity achieved is given by

$$
d^{*}\left(r_{1}, r_{2}, \ldots, r_{K}\right)=\min _{S \subseteq\{1,2, \ldots, K\}: S \neq \emptyset} d_{|S| m, n}^{*}\left(\sum_{k \in S} r_{k}\right),
$$

where $|\cdot|$ for a set denotes the number of elements in the set.

\section{A. Capacity Region and Max-Min Fair Solution}

As mentioned earlier when the queue is nonempty, each user selects a packet uniformly at random from the queue and attempts transmission, with probability $p_{i}, i=A, B$, and independently of the other user; and each user transmits at rate $R_{i}(\mathrm{SNR})=r_{i} \log \mathrm{SNR}, \quad i=A, B$. Let $P_{e}\left(\tilde{R}_{A}(\mathrm{SNR}), \tilde{R}_{B}(\mathrm{SNR})\right)$ denote the probability of decoding error when users transmit at rate $\tilde{R}_{A}(\mathrm{SNR})$ and $\tilde{R}_{B}(\mathrm{SNR})$, respectively at the given value of SNR; the transmission model implies that $\tilde{R}_{i}(\mathrm{SNR}) \in\left\{0, R_{i}(\mathrm{SNR})\right\}, i=A, B$. In the high SNR regime we will also denote the probability of error by $1_{\left[d\left(\tilde{r}_{A}, \tilde{r}_{B}\right)=0\right]}$ where $d\left(\tilde{r}_{A}, \tilde{r}_{B}\right)$ is the diversity gain for transmitting at rates $\tilde{R}_{i}(\mathrm{SNR})=\tilde{r}_{i} \log \mathrm{SNR}$ and $\tilde{r}_{i} \in\left\{0, r_{i}\right\}$ for $i=A, B$ in the system considered.

Our model falls in the general class of models analyzed by Navare, Mergen and Tong [7] and the system capacity region for transmission probability pair $\left(p_{A}, p_{B}\right)$ can be written as

$$
\mathcal{R}^{\mathrm{SNR}}\left(R_{A}(\mathrm{SNR}), R_{B}(\mathrm{SNR}), p_{A}, p_{B}\right)=\alpha \cup \beta,
$$

where

$$
\begin{aligned}
\alpha & =\mathcal{R}_{1}^{\mathrm{SNR}}\left(R_{A}(\mathrm{SNR}), R_{B}(\mathrm{SNR}), p_{A}, p_{B}\right), \\
\beta & =\mathcal{R}_{2}^{\mathrm{SNR}}\left(R_{A}(\mathrm{SNR}), R_{B}(\mathrm{SNR}), p_{A}, p_{B}\right) .
\end{aligned}
$$

Here, $\mathcal{R}_{1}^{\mathrm{SNR}}\left(R_{A}(\mathrm{SNR}), R_{B}(\mathrm{SNR}), p_{A}, p_{B}\right)$ is the set of $\left(\Lambda_{A}(\mathrm{SNR}), \Lambda_{B}(\mathrm{SNR})\right)$ pairs in $\Re_{+}^{2}$ that satisfy

$$
\begin{aligned}
& \Lambda_{B}(\mathrm{SNR})< \\
& \quad R_{B}(\mathrm{SNR})\left[p_{B}\left(1-p_{A}\right)\left(1-P_{e}\left(0, R_{B}(\mathrm{SNR})\right)\right)\right. \\
& \quad+p_{B} p_{A}\left(1-P_{e}\left(R_{A}(\mathrm{SNR}), r_{B}(\mathrm{SNR})\right)\right]=: \bar{R}_{B}(\mathrm{SNR}) \\
& \Lambda_{A}(\mathrm{SNR})< \\
& R_{A}(\mathrm{SNR})\left[p_{A}\left(1-\frac{\Lambda_{B}(\mathrm{SNR})}{\bar{R}_{B}(\mathrm{SNR})}\right)\left(1-P_{e}\left(R_{A}(\mathrm{SNR}), 0\right)\right)\right. \\
& \quad+\frac{\Lambda_{B}(\mathrm{SNR})}{\bar{R}_{B}(\mathrm{SNR})} p_{A}\left(1-p_{B}\right)\left(1-P_{e}\left(R_{A}(\mathrm{SNR}), 0\right)\right) \\
& \left.\quad+\frac{\Lambda_{B}(\mathrm{SNR})}{\bar{R}_{B}(\mathrm{SNR})} p_{A} p_{B}\left(1-P_{e}\left(R_{A}(\mathrm{SNR}), R_{B}(\mathrm{SNR})\right)\right)\right]
\end{aligned}
$$

and $\mathcal{R}_{2}^{\mathrm{SNR}}\left(R_{A}(\mathrm{SNR}), R_{B}(\mathrm{SNR}), p_{A}, p_{B}\right)$ is the set of $\left(\Lambda_{A}(\mathrm{SNR}), \Lambda_{B}(\mathrm{SNR})\right)$ pairs in $\Re_{+}^{2}$ that satisfy

$$
\begin{aligned}
& \Lambda_{A}(\mathrm{SNR})< \\
& \quad R_{A}(\mathrm{SNR})\left[p_{A}\left(1-p_{B}\right)\left(1-P_{e}\left(R_{A}(\mathrm{SNR}), 0\right)\right)\right. \\
& \quad+p_{A} p_{B}\left(1-P_{e}\left(R_{A}(\mathrm{SNR}), R_{B}(\mathrm{SNR})\right)\right)=: \bar{R}_{A}(\mathrm{SNR}) \\
& \Lambda_{B}(\mathrm{SNR})< \\
& R_{B}(\mathrm{SNR})\left[p_{B}\left(1-\frac{\Lambda_{A}(\mathrm{SNR})}{\bar{R}_{A}(\mathrm{SNR})}\right)\left(1-P_{e}\left(0, R_{B}(\mathrm{SNR})\right)\right)\right. \\
& \quad+\frac{\Lambda_{A}(\mathrm{SNR})}{\bar{R}_{A}(\mathrm{SNR})} p_{B}\left(1-p_{A}\right)\left(1-P_{e}\left(0, R_{B}(\mathrm{SNR})\right)\right) \\
& \left.\quad+\frac{\Lambda_{A}(\mathrm{SNR})}{\bar{R}_{A}(\mathrm{SNR})} p_{B} p_{A}\left(1-P_{e}\left(R_{A}(\mathrm{SNR}), R_{B}(\mathrm{SNR})\right)\right)\right]
\end{aligned}
$$


and the net capacity region is

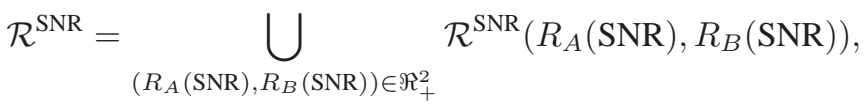

where

$$
\begin{gathered}
\mathcal{R}^{\mathrm{SNR}}\left(R_{A}(\mathrm{SNR}), R_{B}(\mathrm{SNR})\right)= \\
\bigcup_{\left(p_{A}, p_{B}\right) \in[0,1]^{2}} \mathcal{R}^{\mathrm{SNR}}\left(R_{A}(\mathrm{SNR}), R_{B}(\mathrm{SNR}), p_{A}, p_{B}\right) .
\end{gathered}
$$

The physical layer particulars impact the capacity region by the characterization of $P_{e}\left(\tilde{R}_{A}(\mathrm{SNR}), \tilde{R}_{B}(\mathrm{SNR})\right)$. It is easy to argue from this formulation and the symmetry of the problem that the max-min fair operating point is of equal throughput, i.e., $\left(S^{\mathrm{SNR}}(p, R(\mathrm{SNR})), S^{\mathrm{SNR}}(p, R(\mathrm{SNR}))\right)$ and is achieved by setting $p_{A}=p_{B}=p$ and $R_{A}(\mathrm{SNR})=R_{B}(\mathrm{SNR})=R(\mathrm{SNR})$ and thereafter maximizing over allowed values of $p$ and $R$ (SNR). From the discussion above we can directly write

$$
\begin{aligned}
& S^{\mathrm{SNR}}(p, R(\mathrm{SNR}))= \\
& p(1-p) \cdot R(\mathrm{SNR}) \cdot\left(1-P_{e 1}(R(\mathrm{SNR}))\right) \\
& +p^{2} \cdot R(\mathrm{SNR}) \cdot\left(1-P_{e 2}(R(\mathrm{SNR}))\right),
\end{aligned}
$$

where $P_{e 1}(R(\mathrm{SNR}))=P_{e}(R(\mathrm{SNR}), 0)=P_{e}(0, R(\mathrm{SNR}))$ (equality holds by symmetry) and $P_{e 2}(R(\mathrm{SNR}))=$ $P_{e}(R(\mathrm{SNR}), R(\mathrm{SNR}))$. The first term in the above expression equals the probability that exactly one user attempts transmission, multiplied by the rate of transmission and the probability that the transmission is successfully decoded. The second term considers the case when both users transmit and the probability that the transmission is successfully decoded.

In the high SNR regime, the users can pick $\left(R_{A}(\mathrm{SNR}), R_{B}(\mathrm{SNR})\right) \approx\left(r_{A}, r_{B}\right) \log \mathrm{SNR}$ and have $P_{e}^{\mathrm{SNR}}\left(\tilde{r}_{A}, \tilde{r}_{B}\right)$ (for all possible values of $\left(\tilde{r}_{A}, \tilde{r}_{B}\right)$ ) decay to zero, polynomially in SNR, as long as the diversity achieved for the chosen rate pair is positive, i.e., if $d^{*}\left(\tilde{r}_{A}, \tilde{r}_{B}\right)>0$. In this setting,

$$
\begin{array}{r}
\mathcal{R}_{1}^{\mathrm{SNR}}\left(R_{A}(\mathrm{SNR}), R_{B}(\mathrm{SNR}), p_{A}, p_{B}\right) \approx \\
\mathcal{R}_{1}\left(r_{A}, r_{B}, p_{A}, p_{B}\right) \log \mathrm{SNR}
\end{array}
$$

which is given by the set of $\left(\lambda_{A}, \lambda_{B}\right)$ pairs in $\Re_{+}^{2}$ that satisfy

$$
\begin{gathered}
\lambda_{B}<r_{B}\left[p_{B}\left(1-p_{A}\right) 1_{\left[d\left(0, r_{B}\right)>0\right]}\right. \\
\left.+p_{B} p_{A} 1_{\left[d\left(r_{A}, r_{B}\right)>0\right]}\right]=: \bar{r}_{B}, \\
\lambda_{A}<r_{A}\left[p_{A}\left(1-\frac{\lambda_{B}}{\bar{r}_{B}}\right) 1_{\left[d\left(r_{A}, 0\right)>0\right]}\right. \\
+\frac{\lambda_{B}}{\bar{r}_{B}} p_{A}\left(1-p_{B}\right) 1_{\left[d\left(r_{A}, 0\right)>0\right]} \\
\left.+\frac{\lambda_{B}}{\bar{r}_{B}} p_{A} p_{B} 1_{\left[d\left(r_{A}, r_{B}\right)>0\right]}\right],
\end{gathered}
$$

and

$$
\begin{array}{r}
\mathcal{R}_{2}^{\mathrm{SNR}}\left(R_{A}(\mathrm{SNR}), R_{B}(\mathrm{SNR}), p_{A}, p_{B}\right) \approx \\
\mathcal{R}_{2}\left(r_{A}, r_{B}, p_{A}, p_{B}\right) \log \mathrm{SNR},
\end{array}
$$

which is given by the set of $\left(\lambda_{A}, \lambda_{B}\right)$ pairs in $\Re_{+}^{2}$ that satisfy

$$
\begin{aligned}
\lambda_{A}<r_{A}[ & p_{A}\left(1-p_{B}\right) 1_{\left[d\left(r_{A}, 0\right)>0\right]} \\
& \left.+p_{A} p_{B} 1_{\left[d\left(r_{A}, r_{B}\right)>0\right]}\right]=: \bar{r}_{A}, \\
\lambda_{B}<r_{B}[ & p_{B}\left(1-\frac{\lambda_{A}}{\bar{r}_{A}}\right) 1_{\left[d\left(0, r_{B}\right)>0\right]} \\
& +\frac{\lambda_{A}}{\bar{r}_{A}} p_{B}\left(1-p_{A}\right) 1_{\left[d\left(0, r_{B}\right)>0\right]} \\
& \left.+\frac{\lambda_{A}}{\bar{r}_{A}} p_{B} p_{A} 1_{\left[d\left(r_{A}, r_{B}\right)>0\right]}\right],
\end{aligned}
$$

and the net capacity region $\mathcal{R}^{\mathrm{SNR}} \approx \mathcal{R} \log \mathrm{SNR}$ where

$$
\mathcal{R}=\bigcup_{\left(r_{A}, r_{B}\right) \in \Re_{+}^{2}} \mathcal{R}\left(r_{A}, r_{B}\right),
$$

and

$$
\mathcal{R}\left(r_{A}, r_{B}\right)=\bigcup_{\left(p_{A}, p_{B}\right) \in[0,1]^{2}} \mathcal{R}\left(p_{A}, p_{B}\right)
$$

In the Appendix, we plot the achievable rate region of the system for a high value of SNR, with different rates and attempt probabilities, without assuming that the decoding is error-free when the corresponding diversity is positive.

The physical layer particulars now impact the capacity region by the characterization of $1_{\left[d\left(\tilde{r}_{A}, \tilde{r}_{B}\right)>0\right]}$. It is easy to argue from this formulation and the symmetry of the problem that the max-min fair operating point is of equal throughput, i.e., $\left(S^{\mathrm{SNR}}(p, R(\mathrm{SNR})), S^{\mathrm{SNR}}(p, R(\mathrm{SNR}))\right) \approx$ $(S(p, r), S(p, r)) \log \mathrm{SNR}$ and is achieved by setting $p_{A}=$ $p_{B}=p$ and $R_{A}(\mathrm{SNR})=R_{B}(\mathrm{SNR}) \approx r \log \mathrm{SNR}$ and thereafter maximizing over allowed values of $p$ and $r$. The above discussion implies

$$
\begin{aligned}
S(p, r)= & p(1-p) \cdot r \cdot 1_{\left[d_{1}(r)>0\right]}\left(1-P_{e 1}^{\mathrm{SNR}}(r)\right) \\
& +p^{2} \cdot r \cdot 1_{\left[d_{2}(r)>0\right]}\left(1-P_{e 2}^{\mathrm{SNR}}(r)\right),
\end{aligned}
$$

where $d_{1}(r)=d(r, 0)=d(0, r)$ (equality holds by symmetry) and $d_{2}(r)=d(r, r)$.

\section{B. The Joint Decoder Case}

Theorem 1. For a given (large) value of SNR and the input rate $\Lambda$, with the joint decoding of the transmitted messages, the optimal attempt probability for each of the users, $p^{*} \approx 1$ and transmission rate $\approx \min (m, n / 2) \log S N R$ for each user.

Proof: Fix a value of $\Lambda=\lambda \log$ SNR for large, finite SNR, and a rate of transmission $r \log$ SNR for each of the two users. In order to find out the set of rates $\lambda$ that can be supported for the given SNR, we calculate the maximum throughput of the system as follows. Let $d_{1}^{*}(r)$ (resp. $d_{2}^{*}(r)$ ) denote the diversity gain for the system when only one (resp. both) users attempt transmission. In other words, for large SNR, the probability of error at the receiver when only one of the users transmits (resp. both transmit) is upper bounded by $\mathrm{SNR}^{-d_{1}^{*}(r)}$ (resp. $\mathrm{SNR}^{-d_{2}^{*}(r)}$ ). Hence, from Equation (6) 
for large enough SNR, the throughput of the system can be written as

$$
\begin{aligned}
S=S(p, r)= & p(1-p) \cdot r \cdot\left(1-\mathrm{SNR}^{-d_{1}^{*}(r)}\right) \\
& +p^{2} \cdot r \cdot\left(1-\mathrm{SNR}^{-d_{2}^{*}(r)}\right) .
\end{aligned}
$$

Thus, $S$ is a quadratic function of $p$. Maximizing $S$ with respect to $p$, we get

$$
p_{0}=\frac{1-\mathrm{SNR}^{-d_{1}^{*}(r)}}{2\left(\mathrm{SNR}^{-d_{2}^{*}(r)}-\mathrm{SNR}^{-d_{1}^{*}(r)}\right)},
$$

and the optimum attempt probability, which maximizes the throughput $\mathrm{S}$, is given by

$$
p^{*}= \begin{cases}p_{0}, & 0<p_{0}<1 \\ 1, & \text { otherwise }\end{cases}
$$

Now, if $r \geq \min (m, n / 2)$ then $d_{2}^{*}(r)=0$, implying $p_{0}=$ $1 / 2$, exactly. This gives $S_{\max }=r / 4$, where $r$ can at most be $\min (m, n)$. Thus, the maximum throughput per user, if one chooses $r=\min (m, n)-\epsilon$ for some small $\epsilon>0$, is $S_{\text {max }} \approx \frac{1}{4} \min (m, n)$.

If $r=\min (m, n / 2)-\epsilon$ for some small $\epsilon>0$, then for large SNR, the denominator of Equation (7) is small, while the numerator is close to 1 , implying $p_{0} \gg 1$ and $p^{*}=1$, with $S_{\max } \approx \min (m, n / 2)$.

In conclusion, since $2 \min (m, n / 2)>\frac{1}{4} \min (m, n)$ for all positive integers $m, n$, the optimal choices for the transmission rate and the probability of transmission attempt for each user are $(\min (m, n / 2)-\epsilon) \log \mathrm{SNR}$ and 1 , respectively.

\section{The Single User Decoder Case}

In this case, the receiver attempts to separately decode the received message according to the codebook of the individual users. In case of a collision (i.e., both users transmitting simultaneously), the effective SNR for each user's message is $\frac{\text { SNR }}{1+\mathrm{SNR}} \approx 1$, for large SNR. This leads to a situation where a collision implies packet loss. At large SNR, the only term in the throughput expression that survives is the first term - corresponding to only one user transmitting and the other remaining silent. (We note that the throughput expression needs to be more general than Equation (6) to include the terms corresponding to the cases when the receiver decodes only one message after the collision. But this effect can be ignored at high SNR.)

Theorem 2. At high SNR and for the case of single user decoder at the receiver, the optimal probability of transmission attempt is close to $1 / 2$ and the optimal rate of transmission is $\min (m, n) \log S N R$.

Proof: As before, we find the system throughput as a function of the attempt probability, $p$, and each user's rate, $s \log$ SNR for $s>0$. From Equation (6), with $P_{e 2} \approx 1$ for large SNR, we get

$$
S=S(r, p)=p(1-p)\left(1-\mathrm{SNR}^{-d_{1}^{*}(r)}\right) r .
$$

$S$ is maximized at $p=1 / 2$ for any value of $r$. Hence, the maximum value of the throughput $(S)$ is attained by choosing $r=\min (m, n)-\epsilon$ for some $\epsilon>0$, which implies, for large $\mathrm{SNR}$, that $\mathrm{SNR}^{-d_{1}^{*}(r)} \approx 0$ and the maximum throughput per user $\approx \frac{1}{4} \min (m, n) \log$ SNR, with the optimal probability of transmission attempt $=1 / 2$.

As expected, the maximum throughput of the system is lower in the case of single decoder than the case of joint decoder.

The above analysis can be readily extended to the case when there are $K$ non-co-operating users and a single receiver. The system model is almost identical, and the received signal is given by

$$
\mathbf{Y}=\sqrt{\frac{\mathrm{SNR}}{m}} \sum_{i=1}^{K} \mathbf{H}_{i} \mathbf{X}_{i}+\mathbf{W} .
$$

We assume that the block length satisfies $\ell \geq K m+n-1$. In the case of single-user decoding of the transmitted messages, we show that the optimal attempt probability for each user tends to $1 / K$ for SNR $\rightarrow \infty$, and the maximum throughput per user is $\approx \frac{1}{K}\left(\frac{K-1}{K}\right)^{K-1} \min (m, n) \log \mathrm{SNR}$.

Theorem 3. For a given (large) value of SNR and the input rate $\lambda$, with the single-user decoding of the transmitted messages, the optimal attempt probability for each of the users, $p^{*} \approx 1 / K$ and transmission rate $\approx \frac{1}{K} \min (m, n) \log S N R$ for each user.

Proof: The proof of this theorem is very similar to that of Theorem 2. We calculate the system throughput as a function of the attempt probability, $p$, and each user's rate, $r \log$ SNR for $r>0$. Since collision implies loss of all the transmitted packets when SNR is large, the expression for the throughput per user (normalized by $\log \mathrm{SNR}$ ) is

$$
S=S(p, r)=p(1-p)^{K-1}\left(1-\mathrm{SNR}^{-d_{1}^{*}(r)}\right) r .
$$

$S$ is maximized at $p=1 / K$ for any value of $r$. Hence, the maximum value of the throughput $(S)$ is attained by choosing $s=\min (m, n)-\epsilon$ for some $\epsilon>0$, which implies, for large $\mathrm{SNR}$, that $\mathrm{SNR}^{-d_{1}^{*}(r)} \approx 0$ and the maximum throughput per user $\approx \frac{1}{K}\left(\frac{K-1}{K}\right)^{K-1} \min (m, n) \log \mathrm{SNR}$.

\section{Successive Interference Cancellation}

As pointed out by Tse, Viswanath and Zheng in [11], it is possible to achieve a nonzero diversity in the MIMO multiple access channel even when the decoder is not the optimal joint decoder but the successive interference cancellation decoder. With this decoder, the first user's message is decoded treating the other users' interference as noise, its effect is subtracted from the received signal and then the scheme is repeated until all the users' messages are decoded. Failure to decode a user's message at any step will terminate the algorithm, losing all the remaining users' messages. This decoding scheme is better than the single user decoder which, at high SNR, reduces to a collision channel. The performance of this scheme is determined by the first user. From [11], we know that for 
$n \geq K m$ and $\gamma:=n-(K-1) m$, the probability of error for user 1 at high SNR is given by

$$
P_{e 1}^{\prime}(\mathrm{SNR}) \approx \mathrm{SNR}^{-d_{m, \gamma}^{*}(r)} .
$$

Conditioned on the fact that the first user's message is successfully decoded, the second user's message is not decoded with probability

$$
P_{e 2}^{\prime}(\mathrm{SNR}) \approx \mathrm{SNR}^{-d_{m, \delta}^{*}(r)},
$$

where $\delta:=n-(K-2) m$.

Hence, for a two-user system, where each user transmits at a rate $s \log \mathrm{SNR}$, the system throughput can be written as

$$
\begin{aligned}
S_{\text {sys }}= & 2 p(1-p) \cdot r \log \mathrm{SNR} \cdot\left(1-P_{e 1}(\mathrm{SNR})\right)+ \\
& p^{2}\left(\left(1-P_{e 1}^{\prime}\right)\left(2-P_{e 2}^{\prime}(\mathrm{SNR})\right)\right) r \log \mathrm{SNR} .
\end{aligned}
$$

We thus have

$$
\begin{aligned}
\frac{S_{\text {sys }}}{\log \mathrm{SNR}}= & 2 p(1-p) r\left(1-\mathrm{SNR}^{-d_{m, n}^{*}(r)}\right)+ \\
& p^{2} r\left(1-\mathrm{SNR}^{-d_{m, n-m}^{*}(r)}\right)\left(2-\mathrm{SNR}^{-d_{m, n}^{*}(r)}\right) .
\end{aligned}
$$

Remark: We note that $n \geq 2 m$, implying $m \leq n-m$. Hence, the number of transmit antennas is the bottleneck in a sense. At high SNR, the case $p=1, r=m-\epsilon$ for some small $\epsilon>0$ gives $S_{\text {sys }}=2 m \log \mathrm{SNR}$, while $p=1 / 2, r=m-\epsilon$ gives $S_{\text {sys }}=0.5 \mathrm{~m} \log \mathrm{SNR}$. However, one needs to take into account the fact that the diversity for single user detection in case of collision is lower than in the joint decoder case. Hence, although the above expression suggests the optimal operating points to be $(p, r) \approx(1, m)$ for SNR $\rightarrow \infty$, the true optimal operating points at a finite (but reasonably large) SNR will lead to a different choice of $(p, r)$.

\section{The InTERMEdiate SNR REgIME}

Analyzing the maximum throughput of the system for either the joint decoder or single-user decoder for any value of SNR is difficult. We focus on the particular case of $m=1, n=2$, with the single user decoder at the receiver. The transmission scheme considered here is M-QAM. In this case, the received vector is given by

$$
\mathbf{Y}=\sqrt{\operatorname{SNR}}\left(\mathbf{H}_{1} X_{1}+\mathbf{H}_{2} X_{2}\right)+\mathbf{W} .
$$

Now, the system throughput, as given by Equation (6) is $S=2 p(1-p) \cdot R \cdot\left(1-P_{e 1}(R)\right)+2 \cdot p^{2} \cdot R \cdot\left(1-P_{e 2}(R)\right)$.

Here, $1-P_{e 1}(R)$ is the probability that a single user's transmission is successfully decoded. From [4], Section 9.3.1, we know that the probability of bit-error for M-QAM with AWGN is upper-bounded as

$$
P_{b} \leq 2 \exp \left(-\frac{1.5 \mathrm{SNR}}{M-1}\right) .
$$

Relation between $P_{b}$ and $P_{e 1}$ :

If the packet size is $\ell$ symbols, each packet carries $\ell \log M$ bits. Further, assuming $P_{b}$ to be small, we get $P_{e 1}=1-(1-$ $\left.P_{b}\right)^{\ell \log M} \approx(\ell \log M) P_{b}$, implying

$$
P_{e 1} \leq 2 \ell \log M \exp \left(-\frac{1.5 \mathrm{SNR}}{M-1}\right) \text {. }
$$

Analyzing $P_{e 2}: P_{e 2}$ is the probability that a single user's message is successfully decoded in the event of a collision. The receiver attempts to separately decode the message of each transmitter using maximal ratio combining (MRC), which is optimal decoding ([13], Section 3.6.2). In particular, for decoding the message from user 1 , the receiver multiplies the received vector $Y$ with $\mathbf{H}_{1}^{*}$ and, treating interference as noise, decodes the message from user 1 based on the nearest neighbor rule. Assuming $\theta$ to be the angle between the complex vectors $\mathbf{H}_{1}$ and $\mathbf{H}_{2}$ (i.e., $\cos \theta=\frac{\left\|\mathbf{H}_{1}^{*} \mathbf{H}_{2}\right\|}{\left\|\mathbf{H}_{1}\right\| \cdot\left\|\mathbf{H}_{2}\right\|}$ ), the effective SNR seen by the first user is

$$
\begin{aligned}
\mathrm{SNR}_{e f f} & =\frac{E\left(\left\|\mathbf{H}_{1}^{*} \mathbf{H}_{1}\right\|^{2}\left|X_{1}\right|^{2}\right)}{E\left(\left\|\mathbf{H}_{1}^{*} \mathbf{H}_{2}\right\|^{2}\left|X_{2}\right|^{2}\right)+E\left(\| \mathbf{H}_{1}||^{2}\right) E\left(\|\mathbf{W}\|^{2}\right)} \\
& =\frac{\mathrm{SNR} \cdot E\left(\left\|\mathbf{H}_{1}^{*} \mathbf{H}_{1}\right\|^{2}\right)}{\mathrm{SNR} \cdot E\left(\left\|\mathbf{H}_{1}||^{2}\right\| \mathbf{H}_{2} \|^{2} \cos ^{2} \theta\right)+E\left(\left\|\mathbf{H}_{1}\right\|^{2}\right)} .
\end{aligned}
$$

The same effective SNR is seen by user 2 . We thus get an expression for $P_{e 2}$ similar to Equation (10) with SNR replaced by $\mathrm{SNR}_{e f f}$.

For $\theta=\pi / 2$, the channel coefficient vectors $\mathbf{H}_{1}$ and $\mathbf{H}_{2}$ are orthogonal and there is no loss of SNR for any user. The parameter $\theta$ is uniformly distributed on $[0, \pi / 2]$ and for each value of $\theta$, the effective SNR is given by the above expression. The average throughput is thus a function of $\theta$, integrated over the interval $[0, \pi / 2]$ and scaled by the factor $2 / \pi$. Hence, the optimal attempt probability $p$ and the constellation size $M$ (or equivalently, the rate) can be computed numerically, if not in closed form.

\section{CONCLUSION}

In this paper, we considered the general diversitymultiplexing trade-off in multiple access channels under different scenarios: high/intermediate SNR, different types of decoders (single user, successive interference cancellation, joint for all users). We derived expressions for the optimal rates and probabilities of transmission for each user under these models. We showed that these rates and probabilities change depending upon the type of decoder and the SNR range, and in particular, the optimal attempt probability can be strictly between 0 and 1 .

\section{APPENDIX}

We plot the achievable rate region for two user, high SNR case for different values of transmission rate and the probabilities of attempt. Our set up has SNR $=20 \mathrm{~dB}, m=3$ transmit antennas and $n=4$ receive antennas. The achievable region is in general non-convex.

As seen from the plots, when operating near the maximum possible multiplexing gain ( 3 , in this case), the input rate a user can support is heavily dependent on the other user's input rate. The system has maximum throughput when effectively 
only one user is operating. However, at lower values of multiplexing, the sum throughput and maximum of the minimum of the input rates each user can support are both achieved at (or near) the point of unit transmission probability.

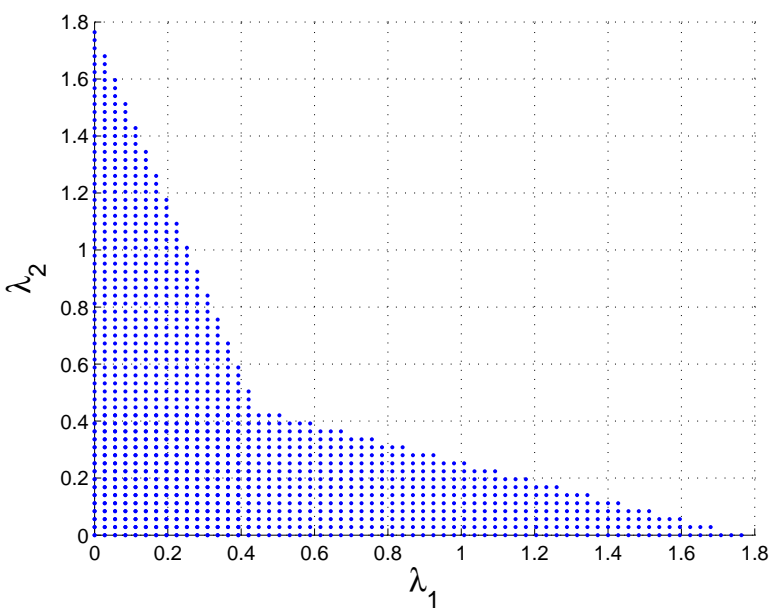

Fig. 2. Achievable Region for $r=2.8, p=0.75$

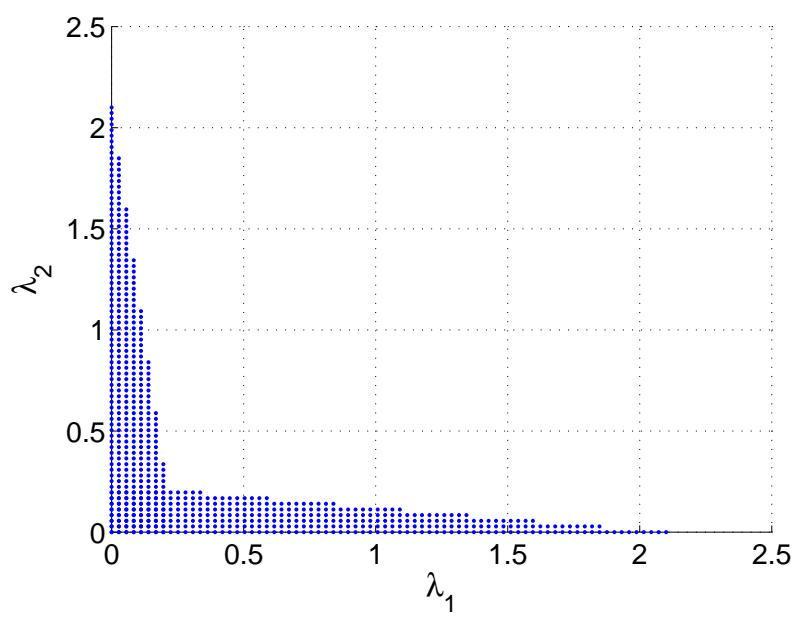

Fig. 3. Achievable Region for $r=2.8, p=0.90$

\section{REFERENCES}

[1] D. Bertsekas and R. Gallager. Data Networks. Prentice-Hall, Englewood Cliffs, NJ, 1987.

[2] Robert G. Gallager. A Perspective on Multiaccess Channels. IEEE Transactions on Information Theory, IT-31(2):124-142, Mar. 1985.

[3] Sylvie Ghez, Sergio Verdú, and Stuart C. Schwartz. Stability Properties of Slotted Aloha with Multipacket Reception Capability. IEEE Transactions on Automatic Control, 33(7):640-649, Jul. 1988.

[4] Andrea Goldsmith. Wireless Communications. Cambridge University Press, 2005.

[5] Jie Luo and Anthony Ephremides. On the Throughput, Capacity, and Stability Regions of Random Multiple Access. IEEE Transactions on Information Theory, 52(6):2593-2607, Jun. 2006.

[6] Muriel Médard, Jianyi Huang, Andrea J. Goldsmith, Sean P. Meyn, and Todd P. Coleman. Capacity of Time-Slotted ALOHA Packetized Multiple-Access Systems Over the AWGN Channel. IEEE Transactions on Wireless Communications, 3(2):486-499, Mar. 2004.

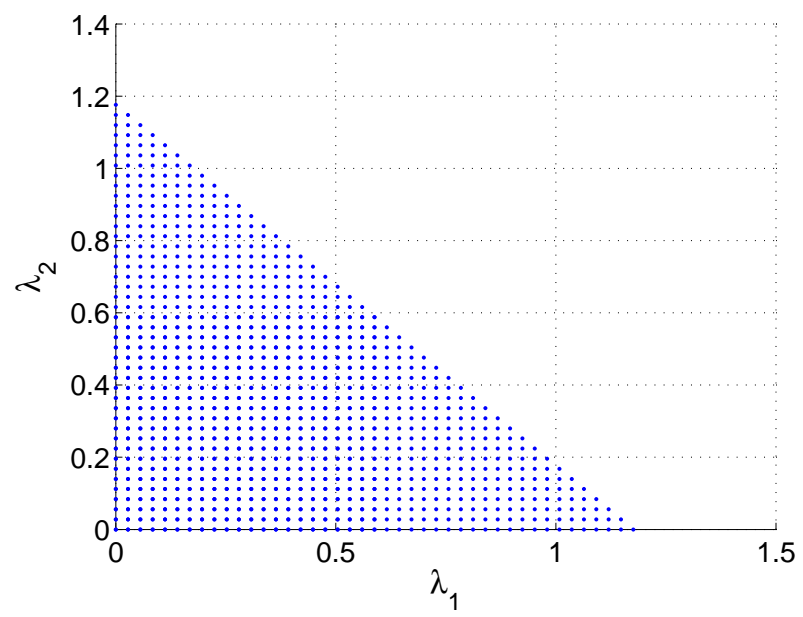

Fig. 4. Achievable Region for $r=2.8, p=0.50$

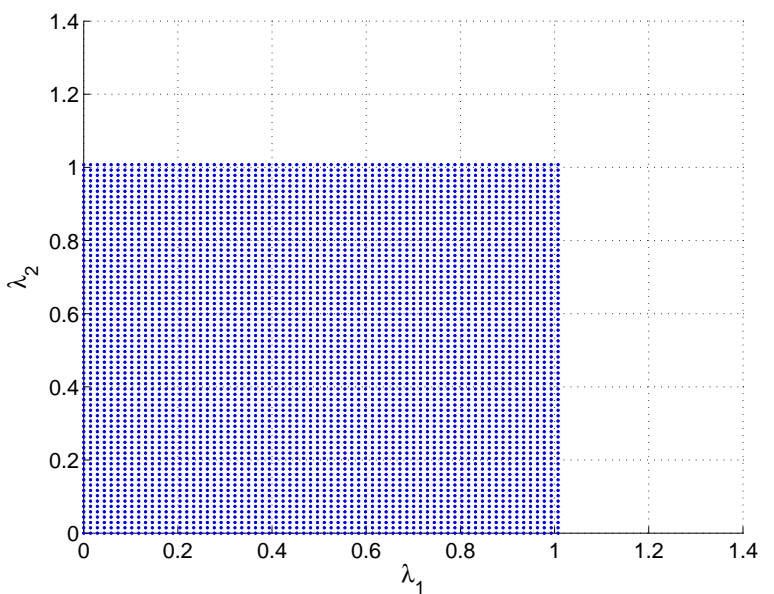

Fig. 5. Achievable Region for $r=1.46, p=0.70$

[7] Vidyut Naware, Gökhan Mergen, and Lang Tong. Stability and Delay of Finite-User Slotted ALOHA With Multipacket Reception. IEEE Transactions on Information Theory, 51(7):2636-2656, Jul. 2005.

[8] Ramesh R. Rao and Anthony Ephremides. On the Stability of Interacting Queues in a Multiple Access System. IEEE Transactions on Information Theory, 34(5):918-930, Sep. 1988.

[9] Junmin Shi and Yi Sun. Random Access Channel with Retransmission Gain. In Military Communications Conference, Oct. 2006.

[10] Brooke Shrader and Anthony Ephremides. Random Access Broadcast: Sstability and Throughput Aanalysis. IEEE Transactions on Information Theory, 53(8):2915-2921, Aug. 2007.

[11] David N. C. Tse, Pramod Viswanath, and Lizhong Zheng. DiversityMultiplexing Tradeoff in Multiple-Access Channels. IEEE Transactions on Information Theory, 50(9):1859-1874, Sep. 2004.

[12] David N. C. Tse and Lizhong Zheng. Diversity and Multiplexing: A Fundamental Tradeoff in Multiple Antenna Channels. IEEE Transactions on Information Theory, 49(5):1073-1096, May 2003.

[13] Sergio Verdú. Multiuser Detection. Cambridge University Press, 2005. 